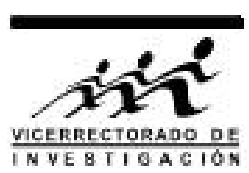

\title{
Procesos físicos que controlan el intercambio de agua entre la Bahía de Puno y la Cuenca Principal del Lago Titicaca, en el Perú.
}

\author{
César Aguirre ${ }^{1}$ \\ ${ }^{1}$ Universidad Nacional Mayor de San Marcos, Ap. Postal 14-0149, Lima, Perú
}

Recibido 15 de junio 2017 - Aceptado 14 agosto 2017

El Lago Titicaca, en la actualidad, es considerado una maravilla natural del mundo. Es el habitad de muchas especies nativas únicas y en extinción, con flora y fauna de gran diversidad, sitio de anidamiento de muchas aves migratorias, fuente de agua dulce filtrada, por su volumen, regula la temperatura del aire de la región permitiendo condiciones del clima menos agresivas para la vida. Sin embargo en las últimas décadas, la influencia antropogénica afectó su conservación. Los problemas que presenta el Lago Titicaca, están concentradas en la zona litoral, las bahías y el Lago Menor. Grandes cantidades de agua residuales que ingresan directamente o deficientemente tratadas, en particular a las Bahías de Puno y Copacabana, están generando procesos de eutrofización. El efecto de las descargas son el incremento de sedimentos acumulados en el fondo, floración explosiva de algas Lemna sp en la superficie, reducción en los niveles de oxígeno disuelto, y disminución de la calidad del agua. Sin embargo, los procesos de contaminación no pueden entenderse solo en función de la carga de materia orgánica que recibe, ya que están bien acoplados a una amplia gama de procesos físicos que van a vincular la dinámica entre la zona litoral y la cuenca principal. Se ha demostrado en grandes lagos, que mueven nutrientes, calor, carbono orgánico, y otros trazadores a través de isóbaras, desde el borde del lago a la plataforma interna, y vice-versa. Estos procesos físicos determinan en gran medida el tiempo promedio en que los contaminantes permanecen en las zonas cercanas a la costa y, por tanto, es necesario estimar su concentración y el nivel de variabilidad en la zona costera. En regiones con fuerte intercambio, los flujos de nutrientes a través de la zona costera parecen estar todavía dominados por grandes entradas de agua, y hay poca evidencia de perturbaciones antropogénicas. En zonas de intercambio restringido, a su vez, existen fuertes evidencias del impacto humano, en términos de cantidad de fitoplancton y abundancia relativa de especies, o zonas profundas del lago donde el oxígeno disminuye. La zona costera del Lago Titicaca, y en particular, la Bahía Interior de Puno, es un ejemplo paradigmático de contaminación causada por la acción humana sin control sobre el entorno cercano a la costa. La Bahía Interior está unido a la Bahía Exterior de Puno por dos canales y una región con mucha totora que genera restricciones al intercambio. Desafortunadamente, poco se sabe sobre el tipo de intercambio o de los procesos físicos que controlan los flujos de sustancias entre la Bahía de Puno y el cuerpo principal del lago. Este, también es el caso de muchas bahías en lagos de todo el mundo, en el que los procesos de intercambio se mantienen en gran parte inexplorados. Por lo tanto, existe una necesidad urgente de comprender los procesos físicos que controlan el intercambio entre zona litoral y pelágico, esto permitiría tratar mejor los problemas de calidad del agua en las zonas costeras. Nuestro objetivo es entender los mecanismos físicos que intervienen en el intercambio de agua y sustancias entre zona litoral y pelágica en grandes lagos tropicales. Utilizamos la Bahía de Puno y el Lago Grande, como un ejemplo del caso. Estimamos el caudal de salida o entrada a través de la boca de la Bahía de Puno, debido a tres procesos físicos que consideramos de mayor importancia que serían los responsables del flujo de agua y sustancias. En particular, se presta atención al intercambio horizontal impulsado por ondas barotrópicas, baroclínicas y procesos de difusión, temas que en la actualidad siguen sin explorarse. Para cumplir con este objetivo se utiliza una combinación de argumentos de escala, basados en datos de campo existentes que apareció principalmente en informes científicos y profesionales, y simulaciones que se realizaron con un modelo de transporte hidrodinámico tridimensional y, originalmente desarrollado por el Servicio Geológico de Estados Unidos, en la Universidad de California, Davis, USA, ampliado y adaptado en la Universidad de Granada para ejecutarse en arquitecturas paralelas. Se estimó los caudales de intercambio y tiempo de residencia de la Bahía de Puno, así como la tasa de difusión para una sustancia, entre el Lago Grande y la Bahía de Puno.

Palabras claves: Ondas barotrópicas, baroclínicas, difusión, tiempo de residencia. 


\section{Physical processes controlling the exchange of water between the Puno Bay and the Main Basin of Lake Titicaca, in Peru.}

Lake Titicaca, at present, is considered a natural wonder of the world. It is the habitat of many unique and endangered native species, with flora and fauna of great diversity, site of nesting of many migratory birds, source of fresh filtered water, by its volume, regulates the air temperature of the region allowing climate conditions less aggressive for life. However, in the last decades, the anthropogenic influence affected its conservation. The problems that Lake Titicaca presents are concentrated in the littoral zone, the bays and the Lake Minor. Large quantities of wastewater that enter directly or poorly treated, in particular to the Bays of Puno and Copacabana, are generating eutrophication processes. The effect of the discharges are the increase of sediments accumulated in the bottom, explosive flowering of Lemna sp algae on the surface, reduction in dissolved oxygen levels, and decrease in water quality. However, pollution processes cannot be understood only in terms of the organic matter load that they receive, since they are well coupled to a wide range of physical processes that will link the dynamics between the littoral zone and the main basin. It has been shown in large lakes, which move nutrients, heat, organic carbon, and other tracers through isobars, from the edge of the lake to the inner shelf, and vice-versa. These physical processes largely determine the average time in which pollutants remain in areas near the coast and, therefore, it is necessary to estimate their concentration and the level of variability in the coastal zone. In regions with strong exchange, nutrient flows through the coastal zone still appear to be dominated by large water intakes, and there is little evidence of anthropogenic disturbance. In areas of restricted exchange, in turn, there is strong evidence of human impact, in terms of the amount of phytoplankton and relative abundance of species, or deep areas of the lake where oxygen decreases. The coastal zone of Lake Titicaca, and in particular, the Interior Bay of Puno, is a paradigmatic example of pollution caused by human action without control over the environment close to the coast. The Interior Bay is linked to the Outer Bay of Puno by two channels and a region with a lot of totora that generates restrictions to the exchange. Unfortunately, little is known about the type of exchange or the physical processes that control the flow of substances between the Bay of Puno and the main body of the lake. This is also the case of many bays in lakes around the world, in which the exchange processes remain largely unexplored. Therefore, there is an urgent need to understand the physical processes that control the exchange between coastal and pelagic zone, this would allow bettering treating water quality problems in coastal areas. Our objective is to understand the physical mechanisms that intervene in the exchange of water and substances between coastal and pelagic zones in large tropical lakes. We use the Bay of Puno and the Big Lake, as an example of the case. We estimate the flow of exit or entry through the mouth of the Bay of Puno, due to three physical processes that we consider of greater importance that would be responsible for the flow of water and substances. In particular, attention is paid to horizontal exchange driven by barotropic waves, baroclinics and diffusion processes, issues that are currently not explored. To fulfill this objective, a combination of scale arguments is used, based on existing field data that appeared mainly in scientific and professional reports, and simulations that were carried out with a three-dimensional hydrodynamic transport model and, originally developed by the Geological Survey Service. United States, at the University of California, Davis, USA, expanded and adapted at the University of Granada to run in parallel architectures. The exchange rates and residence time of the Bay of Puno were estimated, as well as the diffusion rate for a substance, between the Big Lake and the Bay of Puno.

Keywords: Barotropic waves, baroclinic, diffusion, residence time..

\section{Introducción}

Hábitats litorales y pelágicos en grandes lagos tienden a mostrar características físicas, químicas y biológicas, muy diferentes. Las condiciones físicas presentan cambios continuos y muy dinámicos, debido a procesos físicos como absorción de la radiación, evaporación, estratificación, esfuerzo del viento.

Dada la dinámica variable de los hábitats cercanos a la costa, estos son sitios con gran biodiversidad. Las características químicas están relacionadas con los nutrientes inorgánicos necesarios para la vida en el lago. La zona litoral nexo entre las interacciones humanas con los lagos, se han modificado debido a entradas significativas de nutrientes a través de los ríos, como consecuencia de las prácticas agrícolas y ganaderas, así como de aguas residuales de las poblaciones alrededor de los lagos, aumentado dramáticamente la concentración de nutrientes en las últimas décadas.

Hábitats litorales y pelágicos, no se puede entender de forma aislada, están bien acoplados a través de procesos físicos, que determinan en gran medida el tiempo promedio 
en que los contaminantes permanecen en las zonas cercanas a la costa y, por tanto, su concentración y el nivel de deterioro. En zonas de intercambio restringido, existen fuertes evidencias del impacto humano.

En la zona costera del Lago Titicaca, en particular, la Bahía Interior de Puno, la contaminación por altas cargas de materia orgánica y nutriente que entran en la bahía con aguas residuales, conducen a graves problemas de eutrofización y anoxia, [Robert, et al. 2010]. Se han encontrado concentración de clorofila de hasta $25 \mu \mathrm{g} / \mathrm{l}$, típico de lagos eutrófico-a-hipertróficos, en las proximidades de la Bahía Interior de Puno, siendo $12 \mu \mathrm{g} / \mathrm{l}$ el valor máximo para lagos oligotróficos. El crecimiento explosivo de la planta acuática Lemna sp, que cubre la superficie de la Bahía Interior de Puno, es la señal más clara de eutrofización de la Bahía, [Northcote, 1991].

El nivel de eutrofización de la bahía, no puede entenderse sólo en términos de cantidad de nutrientes y carga orgánica. También está estrechamente vinculado al intercambio débil de agua que existe entre la bahía y la cuenca principal del lago [Rueda y Cowen. 2005]. Desafortunadamente, poco se sabe sobre el tipo de intercambio o los procesos físicos que controlan los flujos de material entre zona litoral y pelágico, [Maclntyre y Mellack. 1995; Rao y Schwab. 2007].

Nuestro objetivo es entender los procesos físicos que controlan el intercambio entre zona litoral y pelágica en grandes lagos. Utilizamos la bahía de Puno, como un ejemplo del caso. Cuantificar el papel de los diferentes mecanismos de intercambio entre zona litoral y pelágicos, y analizar el papel de los procesos de intercambio en el desarrollo de la eutrofización de la bahía. En particular, se presta atención al intercambio horizontal de agua y sustancias entre la Cuenca principal y la Bahía de Puno en el Lago Titicaca, impulsado por tres procesos ondas barotrópicas, baroclínicas y difusión. Para cumplir con este objetivo se utiliza una combinación de argumentos de escala, datos de campo existentes que apareció principalmente en informes científicos, así como proponer la recogida de datos de campo adicionales y simulaciones que se llevarán a cabo con un modelo de transporte hidrodinámico tridimensional, SI3D.

\section{2. Área de estudio}

El Lago Titicaca de $895.86 \mathrm{~km}^{3}$ de volumen, está ubicado en América del Sur entre Bolivia y Perú $16^{\circ} \mathrm{S}$. Está formado, al norte por la cuenca principal o Lago Grande de $878.7 \mathrm{~km}^{3}$, de $284 \mathrm{~m}$ de profundidad máxima, al sur el Lago Menor de $12.37 \mathrm{~km}^{3}$, y al oeste la Bahía de Puno de $4.8 \mathrm{~km}^{3}$. La Bahía de Puno, a su vez está formado por la Bahía Interior y Exterior de Puno [D. Wirrmanln. 1991]. Los principales afluentes son: Ramis, Ilave, Coata, Huancané y Suchez, y un efluente el Desaguadero, Fig.1
[Roche et al. 1991]. El nivel del agua del Lago Titicaca ha permanecido casi constante en los últimos cien años, [SENAMHI Puno]. La figura 1 muestra las curvas de nivel de profundidad del Lago Grande conocido como batimetría, obtenido de una carta de sondajes No 650 en metros sobre la base del promedio de 41 años de observaciones, realizado por la Dirección de Hidrografía y Navegación de la Marina de Guerra del Perú DHV y el Servicio de Hidrografía y Navegación Boliviana SHNB, entre los años 1976-1977. El mapa de profundidad tiene información de coordenadas geográficas latitud, longitud y profundidad en la escala de 0:250000. Mayor número de datos de profundidad fueron proporcionados por J. Blanco del trabajo Capacidad de Carga del Lago Titicaca [2011].

\section{Metodología}

Usamos parámetros morfológicos del Lago Titicaca publicados por Wirrmann, 1991, para estimar el Caudal a través de la sección transversal en la boca de la Bahía de Puno, y el Tiempo de Residencia del agua en la Bahía.

Para Ondas Barotrópicas que se desplazan en aguas poco profundas, producidas por el torque del viento, estimamos la rapidez de la onda $C_{0}$ en función de la profundidad media de la Bahía de Puno H y la aceleración de la gravedad (g) [Barreiro, 2012]. El caudal Q fue estimado como la rapidez (u) del agua en dirección hacia la Bahía de Puno por la sección transversal en la boca de la Bahía ( $a^{*} Z$ ), obtenidos del resultado de la simulación realizada en el verano del 2011, y del de mapa de batimetría. El tiempo de residencia (TR) del agua en la Bahía de Puno, fue estimado de la relación del volumen $V$ de la Bahía de Puno, donde $\mathrm{A}$ es el ancho medio, $\mathrm{L}$ el largo y $\mathrm{H}$ la profundidad media de la Bahía y el caudal Q, Fig. 2 [Wirrman, 1991].

$$
\begin{gathered}
C_{0}=\sqrt{g H} \\
V=A * H * L \\
Q=a * Z * u \\
T=\frac{\lambda}{C_{0}}=\frac{4 L}{C_{0}} \\
T R=\frac{V}{Q}
\end{gathered}
$$

Para las Ondas Baroclínicas producidas por fuerzas restauradoras gravitatorias, en aguas profundas, estimamos la rapidez de la onda $\left(C_{0}\right)$ considerando la gravedad reducida $\left(g^{\prime}\right)$, para un modelo del lago de dos capas de espesores h1 y h2. Para estimar $g^{\prime}$ se consideró el coeficiente de dilatación cúbica del agua $\beta=2.1 \times 10^{-4}{ }^{\circ} C^{-1}$, el gradiente 


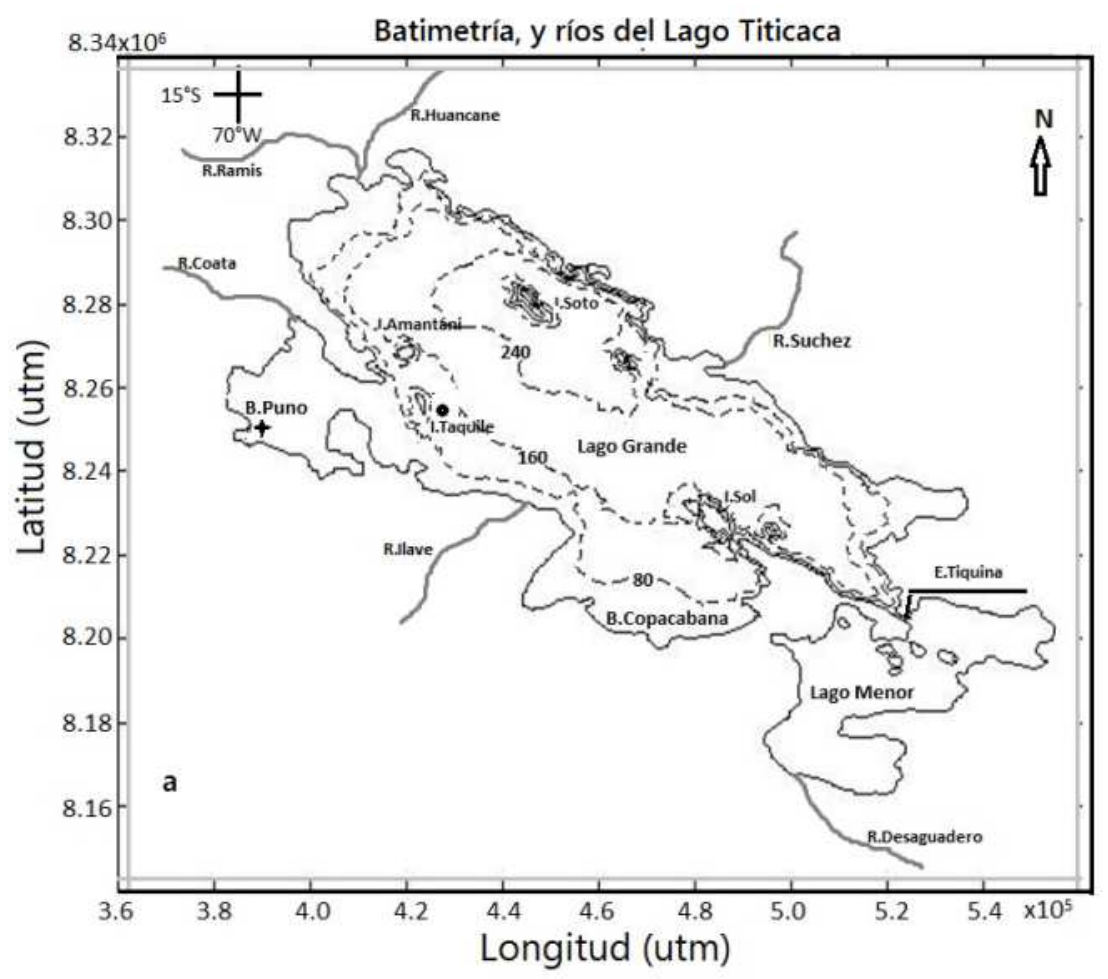

Figura 1: El Lago Titicaca, constituido por las Bahías de Puno y Copacabana. Al norte el Lago Grande, al sur el Lago Menor. Principales islas: Amantani, Soto, Taquile y Sol. Principales afluentes: Ramis, llave, Coata, Huancané y Suchez, y un efluente Desaguadero. La batimetría del Lago Grande presenta la profundidad máxima cerca de la isla Soto.

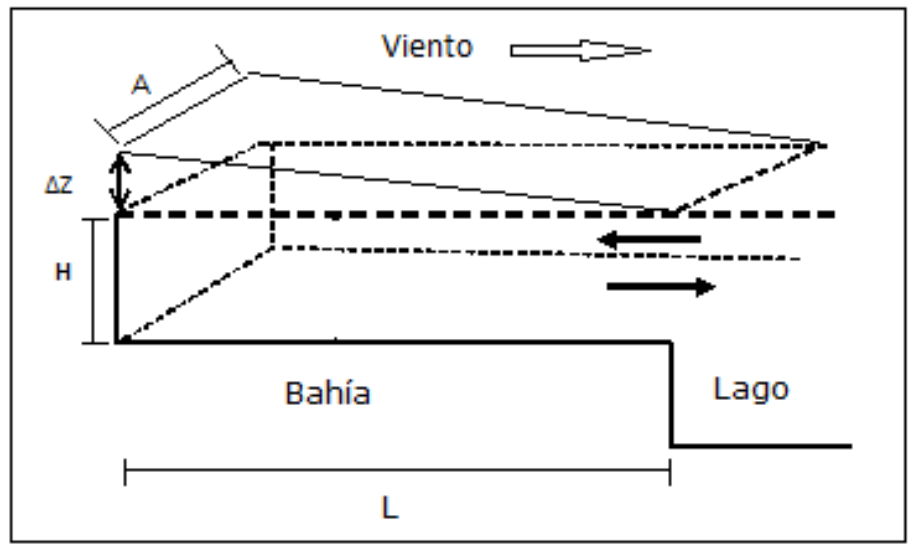

Figura 2: Modelo de la Bahía de Puno y Lago Grande. Onda Barotrópica, impulsando intercambio de agua entre la Bahía de Puno y Lago Grande. 
de temperatura $\Delta T=3^{\circ} \mathrm{C}$ obtenido de simulaciones realizadas con un modelo hidrodinámico SI3D, y de perfiles de temperatura del agua (Figura 3 ).

$$
\begin{gathered}
C_{0}=\sqrt{\frac{g^{\prime} h_{1} h_{2}}{H}} \\
g^{\prime}=g \beta \Delta T
\end{gathered}
$$

El periodo $T$ de la onda, se estimó de la relación entre el perímetro $\mathrm{P}$ del Lago Grande y la rapidez de la Onda Baroclínica $C_{0}$.

$$
T=\frac{P}{C_{0}}
$$

Los resultados de la simulación mostraron la elevación de la interfase en la boca de la Bahía de Puno (h) debido al esfuerzo del viento. Estimamos el caudal $Q$ como la rapidez del agua (u) en la dirección hacia la Bahía de Puno por el área de la sección transversal (ah), en la boca de la Bahía, Fig. 4.

$$
Q=a * h * u
$$

El tiempo de residencia (TR) estimado para la bahía de Puno debido a este proceso es

$$
T R=\frac{V}{Q}
$$

Donde $\mathrm{V}$ es el volumen de la Bahía de Puno y $\mathrm{Q}$ el caudal estimado para el intercambio por Ondas Baroclínicas.

En el proceso de Difusión, se estimó la tasa de intercambio entre Bahía de Puno y el Lago Grande, como el flujo efectivo de sustancias a través de la sección transversal de la Bahía de Puno, en función de las concentración de sustancias en el Lago Grande $C_{1}$ y la Bahía de Puno $\mathrm{C}_{2}$. Considerando que se conserva la masa de la sustancia y los sistemas están mezclados parcialmente, podemos expresar el balance de masa como:

$$
\begin{aligned}
& v_{1} \frac{d C_{1}}{d t}=F_{1}-Q_{1} C_{1}-K_{1} V_{1} C_{1}+E^{\prime}\left(C_{2}-C_{1}\right) \\
& v_{2} \frac{d C_{2}}{d t}=F_{2}-Q_{1} C_{2}-K_{2} V_{2} C_{2}+E^{\prime}\left(C_{2}-C_{1}\right)
\end{aligned}
$$

El primer término es la variación de masa de la sustancia en el tiempo. En el segundo término, $\mathrm{F}$ es la carga total que ingresa, QC flujo de salida (-) y entrada (+), KVC perdida por reacciones químicas o sedimentación, y $E^{\prime}\left(C_{2}-C_{1}\right)$ movimiento por difusión, los subíndices 1,2 corresponden a la región profunda y somera respectivamente, Fig. 6.

Considerando que la masa de la sustancia se conserva, podemos escribir $v_{2} \frac{d S_{2}}{d t}=F_{2}-Q_{2} S_{2}-K_{2} V_{2} C_{2}+Q_{2} C_{2}+E^{\prime}\left(S_{1}-S_{2}\right)$

Donde $\mathrm{S}$ es la concentración de la sustancia conservativa. Para el estado estacionario tenemos

$$
E_{\prime}^{\prime}=\frac{F_{2}-Q_{2} S_{2}}{S_{2}-S_{1}}
$$

Donde $E_{\prime}$ es la tasa de difusión efectiva de la sustancia a través de la sección transversal de la bahía. Estimaremos el valor de E' para datos del Lago Grande y Bahía de Puno, usando la ecuación [14].

\section{Resultados}

Intercambio de agua impulsado por Ondas Barotrópicas

Estimamos los valores de rapidez de la onda Co, volumen total $V$, el caudal $Q$, y el tiempo de residencia $T R$, a partir de las ecuaciones 1 , 2, 3, 4, y 5 . Los datos morfológicos son de publicaciones realizadas por Wirmann en 1991, y de simulaciones realizadas en el verano del 2011. Los resultados se muestran en la tabla 1 .

Intercambio de agua impulsado por Ondas Baroclínicas

Se estimó la gravedad reducida $g^{\prime}$, la rapidez de la onda $C_{0}$, y del agua (u) en la dirección hacia la Bahía de Puno, el caudal $Q$, y el tiempo de residencia TR. Los datos morfológicos son de publicaciones realizadas por Wirrmann en 1991, y de simulaciones realizadas en el verano del 2011. En el verano la columna de agua estuvo estratificada, con la termoclina a $50 \mathrm{~m}$ de profundidad, intensidad media del viento de $5 \mathrm{~m} / \mathrm{s}$ y ráfagas de 11 $\mathrm{m} / \mathrm{s}$, dirección preferencial de oeste a este por periodos de 12 a 24 h. Las estimaciones del Caudal Q y Tiempo de Residencia TR, se presentan en la tabla 2.

\section{Intercambio de sustancias impulsado por Difusión.}

Se estimó el flujo de fosforo a través de la boca de la Bahía de Puno. Considerando valores referenciales de fósforo en la Bahía de Puno y Lago Grande [Chapra, 1997]. Estimamos la tasa de difusión de fósforo sin sedimentación, ecuación [14]. Considerando la sedimentación de fósforo suspendido en la bahía, la ecuación [14] se modifica a:

$$
\begin{gathered}
E_{\prime}^{\prime}=\frac{F_{2}-Q_{2} S_{2}-F_{s}}{S_{2}-S_{1}} \\
F_{s}=V_{s} * A_{s} * S_{2}
\end{gathered}
$$

Donde, $F_{s}$ es la sedimentación de fósforo, Vs es velocidad de sedimentación estimado a partir de la ley de Stokes 


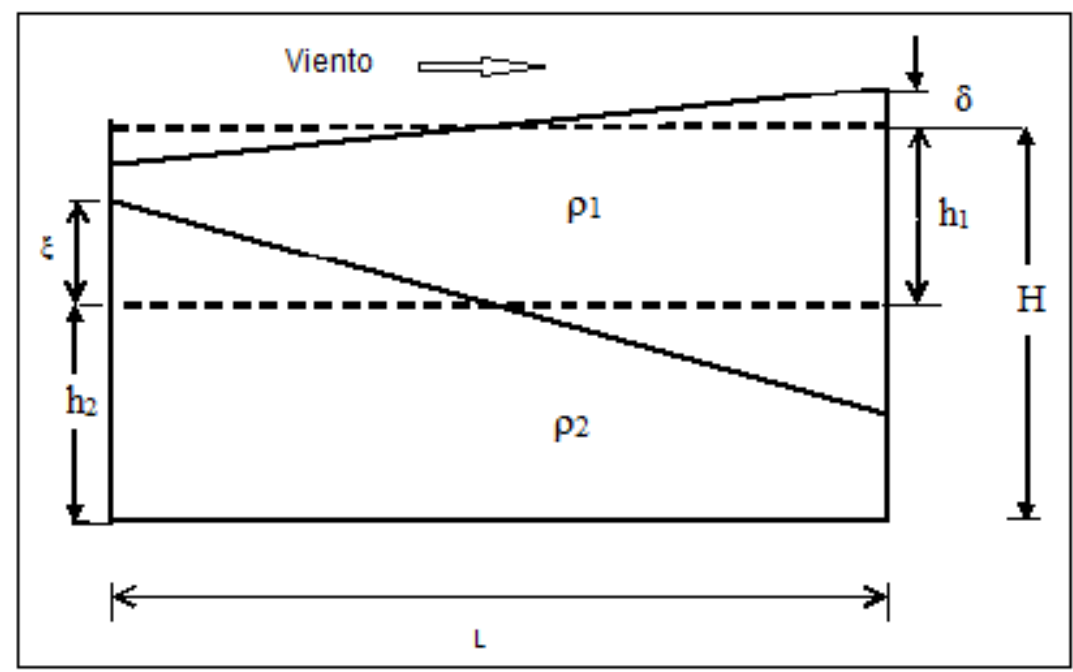

Figura 3: Modelo de dos capas del Lago Grande, de densidades $\rho_{1}$ y $\rho_{2}$. Espesores de las capas $h_{1}$ y $h_{2}, \mathrm{H}$ la profundidad media del Lago Grande y $\delta$ elevación del nivel del agua en la costa por donde sale el viento, que genera inclinación de la interfase.

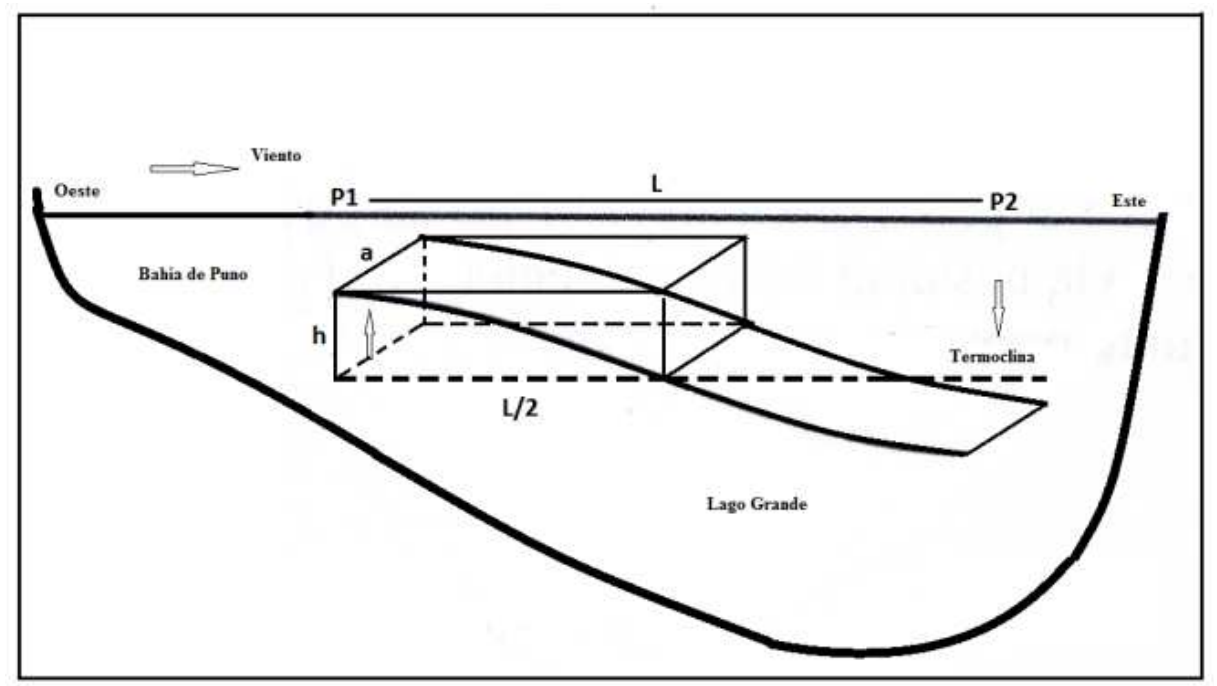

Figura 4: Modelo de Ondas Baroclínicas elevándose en la boca de la Bahía de Puno, debido a la acción del viento que acumula agua en la costa por donde sale. 


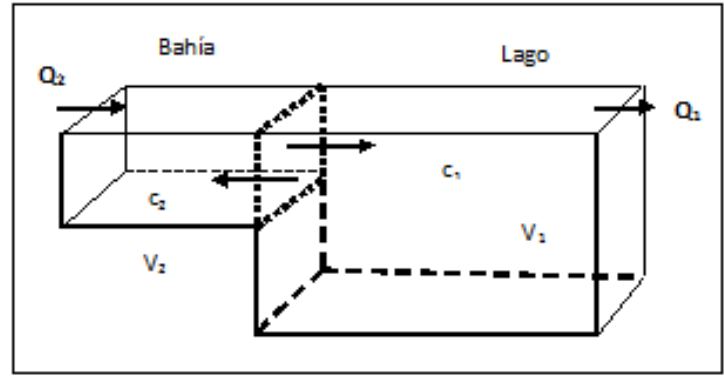

Figura 5: Desplazamiento de sustancias de regiones de mayor a menor concentración. Considerando $C_{1}$ y $C_{2}$ concentraciones de una sustancia en Lago Grande y Bahía de Puno, de volúmenes $V_{1}$ y $V_{2}$, tal que $C_{2}>C_{1}$.

\begin{tabular}{lcr}
\hline Parámetros & Valor & Unidad \\
\hline Rapidez de la onda $\left(C_{0}\right)$ & 8.6 & $\mathrm{~ms}^{-1}$ \\
Profundidad media $(\mathrm{H})$ & 8 & $\mathrm{~m}$ \\
Longitud (L) & 41000 & $\mathrm{~m}$ \\
Ancho medio (A) & 14000 & $\mathrm{~m}$ \\
Elevación media $(\Delta Z)$ & 0.01 & $\mathrm{~m}$ \\
Volumen (V) & $4.6 \times 10^{9}$ & $\mathrm{~m}^{3}$ \\
Periodo de la onda (T) & $19.1 \times 10^{3}$ & $\mathrm{~s}$ \\
Ancho boca BP (a) & 6700 & $\mathrm{~m}$ \\
Rapidez del agua (u) & $1.1 \times 10^{-2}$ & $\mathrm{~ms}^{-1}$ \\
Profundidad boca (z) & 40 & $\mathrm{~m}$ \\
Caudal (Q) & $2.9 \times 10^{3}$ & $\mathrm{~m}^{3} \mathrm{~s}^{-1}$ \\
Tiempo Residencia(TR) & 18.5 & días \\
\hline
\end{tabular}

Tabla 1: Parámetros de la Bahía de Puno en el intercambio impulsado por Ondas Barotrópicas. Estimación del Caudal y Tiempo de Residencia.

\begin{tabular}{||l|lr||}
\hline Parámetros & Valor & Unidad \\
\hline Gravedad reducida & 0.0062 & $\mathrm{~ms}^{-2}$ \\
Profundidad $\left(h_{1}\right)$ & 50 & $\mathrm{~m}$ \\
Profundidad $\left(h_{2}\right)$ & 85 & $\mathrm{~m}$ \\
Profundidad (H) & 135 & $\mathrm{~m}$ \\
Rapidez $\left(C_{0}\right)$ & 0.44 & $\mathrm{~ms}^{-1}$ \\
Perimetro (P) & $4.55 \times 10^{5}$ & $\mathrm{~m}$ \\
Periodo (T) & $10.3 \times 10^{5}$ & $\mathrm{~s}$ \\
Volumen (V) & $4.6 \times 10^{9}$ & $\mathrm{~ms}$ \\
Ancho medio (a) & 6700 & $\mathrm{~m}$ \\
Amplitud (h) & 40 & $\mathrm{~m}$ \\
Rapidez agua (u) & $0.85 \times 10^{-2}$ & $\mathrm{~ms}^{-1}$ \\
Caudal (Q) & $2.3 \times 10^{3}$ & $\mathrm{~m}^{3} \mathrm{~s}^{-1}$ \\
Tiempo Residencia(TR) & 23.5 & $\mathrm{días}$
\end{tabular}

Tabla 2: Parámetros de la Bahía de Puno en el intercambio impulsado por Ondas Barotrópicas. Estimación del Caudal y Tiempo de Residencia.

\begin{tabular}{lcr}
\hline Parámetros & Valor & Unidad \\
\hline Bahía de Puno & & \\
Volumen $\left(V_{2}\right)$ & $4.6 \times 10^{9}$ & $\mathrm{~m}^{3}$ \\
Profundidad $(H 2)$ & 8 & $\mathrm{~m}$ \\
Area superficial $\left(A_{2}\right)$ & $1.1 \times 10^{9}$ & $\mathrm{~m}^{2}$ \\
Flujo de salida $\left(Q_{2}\right)$ & 0.560 & $\mathrm{~m}^{3}$ \\
Concentración $\left(S_{2}\right)$ & & \\
Bahía de Puno & $878.7 \times 10^{-1}$ & $\mathrm{~m}^{3}$ \\
Volumen $\left(V_{1}\right)$ & 135 & $\mathrm{~m}$ \\
Profundidad $\left(h_{1}\right)$ & $649 \times 10^{6}$ & $\mathrm{~m}^{2}$ \\
Area superficial $\left(A_{1}\right)$ & $4.4 \times 10^{9}$ & $\mathrm{~m}^{3} \mathrm{ao}^{-1}$ \\
Flujo de entrada $\left(Q_{1}\right)$ & 0.008 & $\mathrm{gm}^{-3}$ \\
Concentración $\left(S_{1}\right)$ & $2.47 \times 10^{2}$ & \\
Tasa de difusión sin sedimentación $\left(E^{\prime}\right)$ & 1.64 & $\mathrm{md}^{-1}$ \\
Velocidad Sedi. $\left(V_{s}\right)$ & $2 \times 10^{6}$ & $\mathrm{~m}^{2}$ \\
Area sedimentacion $\left(A_{s}\right)$ & $2.8 \times 10^{2}$ & $\mathrm{~m}^{3} \mathrm{~S}^{-1}$ \\
Tasa de difusion con sedimentacion $\left(E_{\prime}\right)$ & &
\end{tabular}

Tabla 3: Parámetros de la Bahía de Puno y Lago Grande en el intercambio de sustancia impulsado por difusión. Estimación de la Tasa de Difusión. 
considerando un flujo laminar, As el área de sedimentación y $S_{2}$ la concentración de fósforo en la Bahía de Puno. Las estimaciones de las Tasas de Difusión se presentan en la tabla3.

\section{Discusión}

El Caudal y el Tiempo de Residencia estimado para los procesos que impulsan el intercambio de agua por Ondas Barotrópicas y Baroclínicas presentan valores cercanos, pero indican que el proceso por Ondas Barotrópicas sería más eficiente para remover el agua de la Bahía de Puno. Mientras que la Tasa de Difusión de fósforo de la Bahía de Puno hacia el Lago Grande, presentan valores menores que los caudales de los procesos anteriores. No es posible presentar porcentajes de incertidumbre en las estimaciones debido a que no se dispone de medidas de Caudal y Tasa de Difusión en la boca de la Bahía de Puno. Es un primer intento de cuantificar estos parámetros en base a datos existentes y datos simulados.

\section{Conclusiones}

Considerando los valores del Caudal y Tiempo de Residencia estimadas para un intercambio de agua entre la Bahía de Puno y el Lago Grande, podemos concluir que se tardaría en remplazar el agua de la Bahía de Puno, por el primer y segundo proceso 18.5 y 23.1 días respectivamente, considerando las mismas condiciones, es decir que el caudal sea constante, situación no muy real. Respecto al proceso de difusión, es notable la diferencia en la Tasa de Difusión si no se considera la sedimentación. El método implementado deber ser mejorado con datos medidos de modo que las estimaciones sean más confiables y entender mejor la dinámica del sistema.

La Bahía Interior de Puno presenta un nivel alto de contaminación, cuyo intercambio de agua con la Bahía Exterior es más débil aún. Se podría cuantificar los caudales de intercambio por este método, y ajustar los resultados comparando con datos de velocidad de la corriente medidos en los dos canales de ingreso. Estos resultados podrían convencer a los que desean resolver el problema de contaminación de la Bahía Interior de Puno, sosteniendo que el sistema tiene un tiempo de renovación del agua. El primer paso debe ser restringir la descarga de agua residual sin tratamiento a la Bahía Interior de Puno.

\section{Referencias}

[1] J. Blanco. Desafíos para el modelamiento de la capacidad de carga. Simposio International "El Estado del Lago Titicaca: Desafíos para una Gestión basada en el Ecosistema" Puno - Perú, 19 al 21 de octubre del 2011.

[2] M. Barreiro. Notas de clase Cap. 7.(2012) http://meteo.fisica.edu.uy/Materias

[3] S. Chapra. Texto: Surface Water-Quality Modeling. Copyright Waveland Press, Inc. (1997).

[4] R. Rao, and D. Schwab. Transport and Mixing Between the Coastal and Offshore Waters in the Great Lakes: a Review, J. Great Lakes Res. 33:202-218 Internat. Assoc. Great Lakes Res. (2007).

[5] F.J.Rueda, and A. Cowen. Residence time of a freshwater embayment connected to a large lake. Limnol. Oceanogr., 50(5), 1638-1653.(2005).

[6] S. Maclntyre, and J. Melack. Vertical and horizontal transport in lakes linking littoral, benthic and pelagic habitats. J. North Am. Benthol. Soc., 14: 599 615.(1995).

[7] P. Roberts, B. Villegas. Water Quality in Lake Titicaca. Prepared for the World Bank.(2007)

[8] T. Northcote (1991). Eutrofización y problemas de polución. El Lago Titicaca Síntesis del conocimiento limnológico actual. Texto. pp 563-572. (1991).

[9] M. Roche, J. Bouges, J. Cortes, R.Mattos. Climatology and hidrology of the Lake Titicaca basin. El Lago Titicaca Síntesis del conocimiento limnológico actual. Texto. pp 63-87. (1991).

[10] D. Wirmann. Morfología y Batimetría. El Lago Titicaca Síntesis del conocimiento limnológico actual. Texto. pp 31-37. (1991). 